\title{
Measurement of the In-Plane Thermal Conductivity of Long Fiber Composites by Inverse Analysis
}

\author{
Bassam Assaf, Vincent Sobotka, François Trochu \\ Applied Research Center on Polymer (CRASP), Department of Mechanical Engineering, Ecole Polytechnique de Montréal, \\ Station "Centre-Ville", Montreal, Canada \\ Email: bassam.assaf@polymtl.ca
}

How to cite this paper: Assaf, B., Sobotka, V. and Trochu, F. (2017) Measurement of the In-Plane Thermal Conductivity of Long Fiber Composites by Inverse Analysis. Open Journal of Composite Materials, 7, 85-98. https://doi.org/10.4236/ojcm.2017.72005

Received: February 18, 2017

Accepted: April 27, 2017

Published: April 30, 2017

Copyright @ 2017 by authors and Scientific Research Publishing Inc. This work is licensed under the Creative Commons Attribution International License (CC BY 4.0).

http://creativecommons.org/licenses/by/4.0/

(c) (i) Open Access

\begin{abstract}
In the present work, inverse thermal analysis of heat conduction is carried out to estimate the in-plane thermal conductivity of composites. Numerical simulations were performed to determine the optimal configuration of the heating system to ensure a unidirectional heat transfer in the composite sample. Composite plates made of unsaturated polyester resin and unidirectional glass fibers were fabricated by injection to validate the methodology. A heating and cooling cycle is applied at the bottom and top surfaces of the sample. The thermal conductivity can be deduced from transient temperature measurements given by thermocouples positioned at three chosen locations along the fibers direction. The inverse analysis algorithm is initiated by solving the direct problem defined by the one-dimensional transient heat conduction equation using a first estimate of thermal conductivity. The integral in time of the square distance between the measured and predicted values is the criterion minimized in the inverse analysis algorithm. Finally, the evolution of the in-plane composite thermal conductivity can be deduced from the experimental results by the rule of mixture.
\end{abstract}

\section{Keywords}

Inverse Analysis, In-Plane Thermal Conductivity, Mold Design

\section{Introduction}

Resin Transfer Molding (RTM) has become in recent years a more widely adopted process to manufacture automotive and aerospace composite parts. A good thermal control of the process is required to increase process efficiency and improve part quality. Temperature during mold filling and cure of the compo- 
site plays an important role in determining the final properties of the parts. It is therefore important to analyze the thermal behavior during processing and hence evaluate the influence of temperature on thermal properties such as specific heat and thermal conductivity.

While specific heat can be obtained from DSC measurements, thermal conductivity is more difficult to measure, especially for anisotropic materials like composites. Several methods exist, all based on conductive heat transfer. They consist of thermally exciting a sample and measuring its thermal response in order to estimate its heat transport properties. To avoid coupling heat conduction with any other phenomenon, the characterization methods are conducted, most of the time, independently from the cross-linking phase of the polymer resin. An appropriate model can be used afterwards to describe the dependence of thermal conductivity with the degree of cure.

Since composites usually exhibit anisotropic material properties, knowledge of in-plane thermal conductivity plays also an important role in many applications. As for the through-thickness heat conductivity of composites, a new approach is also needed to measure the in-plane thermal conductivity as a function of temperature. In a previous paper [1], inverse thermal analysis was applied to measure the transverse heat conductivity of composites in a mold especially devised for that purpose. This technique will be further enhanced here by applying these ideas in a simple and novel way to measure the in-plane heat conductivity of composite specimens. The main originality of our approach stems from the way the composite samples are prepared so that the experiments required for inverse thermal analysis can be carried out in the same test mold devised in [1] for through-thickness heat conductivity measurements.

Inverse methodology has been widely used in thermal analysis along with several other experimental procedures [2] [3]. An approach based on inverse thermal analysis allows identifying unknown parameters of interest from experimental measurements. As a matter of fact, when the explicit relationship between the measured data and the state parameters is not obvious, inverse thermal analysis provides a unique and effective way to estimate physical parameters of interest.

This paper complements work published in [1] on the measurement of the composite transverse thermal conductivity in a mold especially devised for that purpose. The test mold was described thoroughly in this previous work, which explains how it was designed to create a uniform through-thickness heat flow through composite specimens and how inverse thermal analysis can be implemented to measure the composite through-thickness thermal conductivity. The present article will show how the same mold can be used successfully to measure the in-plane composite thermal conductivity, if the composite samples are properly prepared and the experiment is carried out in as described in the sequel.

The in-plane heat conductivity is obtained from temperature measurements carried out along the in-plane direction of composite specimens made of an unsaturated polyester resin and unidirectional glass fibers. After a review of rele- 
vant bibliography, the test mold and the experimental procedure are briefly recalled, followed by a detailed description of the experimental protocol. Finally, experimental results for in-plane measurements are presented for a cured composite specimen and compared to an analytical model. The main features of thermal inverse methodology are summed up in Appendix.

\section{Bibliography}

Classical techniques to measure thermal conductivity may be regrouped in two main categories, namely steady-state and transient techniques [4] [5]. Steadystate approaches are usually based on parallel plate techniques such as the guarded hot plate [6] or guarded heat flow meter [7]. In these methods, by setting different constant temperatures on two opposite surfaces of the specimens, the sample is submitted to a one-dimensional conductive heat flux through its thickness [6]. One of the main drawbacks in the case of low diffusivity materials is the time necessary to reach steady-state.

Transient techniques such as hot wire [8], flash method [9] [10], transient plane source [11] [12] or periodic methods [13] [14] allow a more rapid estimation provided that the isothermal state is reached before the experiment is performed. The models describing heat transfer in these experimental tests are more complex than those of the previous methods. In addition, knowledge of the specific heat and density is often required. Nevertheless, both families of methods present the same drawbacks:

- Each measurement is performed at a given temperature, hence requiring multiple tests to characterize the heat conductivity as a function of temperature.

- Most of the above-mentioned approaches are only suited to carry out through-thickness heat conductivity measurements.

The thermal conductivity measured by this experimental approach is a macroscopic value averaged on the size of the composite specimen, namely for the number of reference elementary volumes contained in the tested sample. This macroscopic parameter is the information needed in non-isothermal process simulations of resin injection such as in a software like PAM-RTM [15]. The microstructural values of thermal conductivity would still be of interest for more detailed analyses. As for the permeability of fibrous reinforcements, the microscopic heat conductivity could also be derived from analytical solutions for regularly packed unidirectional fibers [16].

\section{Manufacturing of Composite Specimens}

\subsection{Mold Description}

As shown in Figure 1, the mold is composed of two main parts. It was designed to produce composite planar parts of different thicknesses by Resin Transfer Molding (RTM). The height of the cavity where the resin is injected can be adjusted with the mobile bottom plate where four screws positioned at its corners 

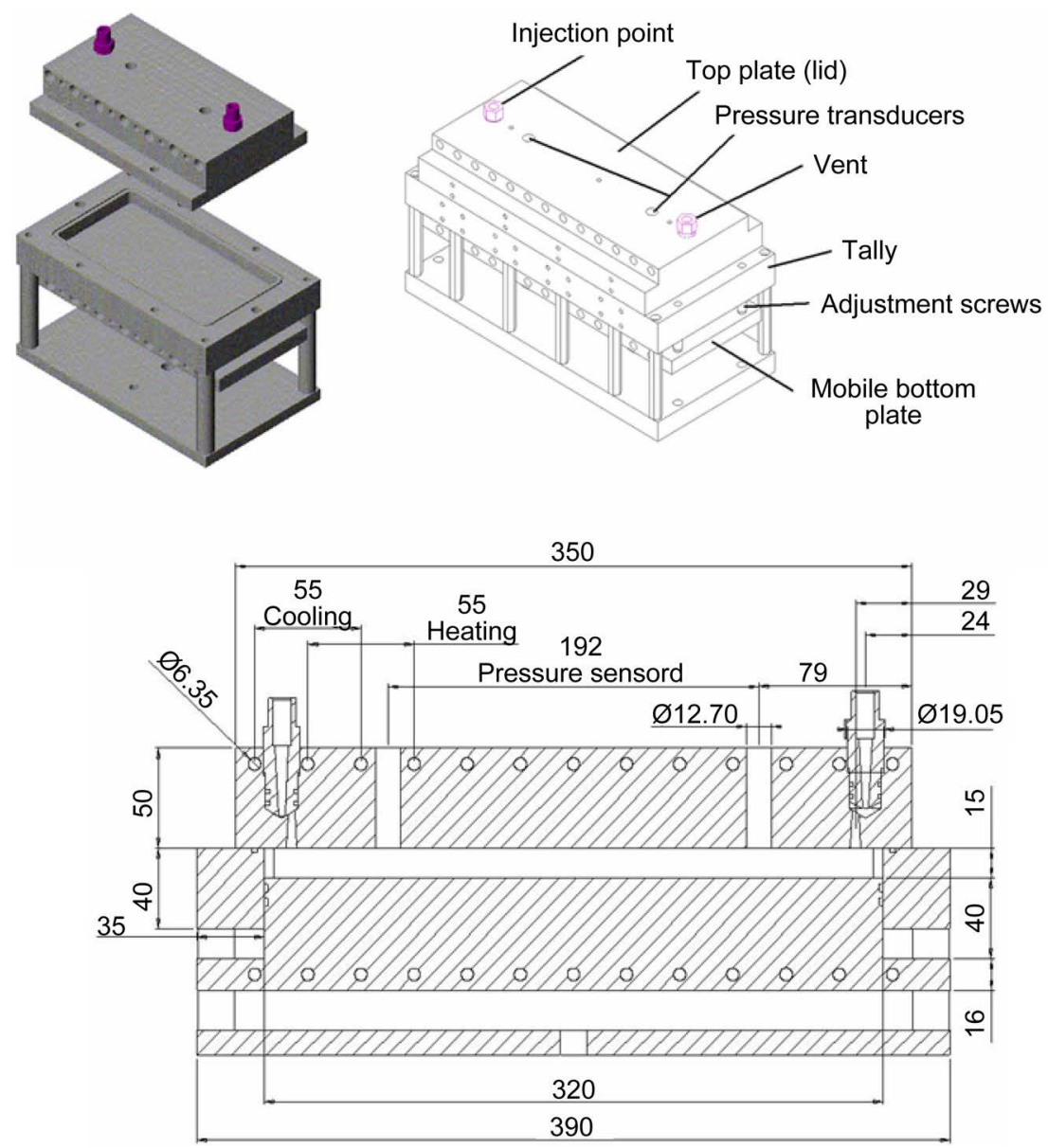

Figure 1. Perspective view and cross-section of the test mold with its geometric dimensions.

allow controlling the fiber volume content. Two other screws located on the top surface of the mold are used as injection port and vent. Figure 1 shows also a vertical cross-section of the mold. The length and width of the cavity are 320 $\mathrm{mm}$ and $110 \mathrm{~mm}$ respectively, while the maximum cavity thickness allowed by the mobile plate is $35 \mathrm{~mm}$. A rubber seal is placed between the cover and the base plate to avoid any leakage of the resin. The mold is made of aluminum. It can be heated or cooled uniformly and resists to processing temperatures up to $200^{\circ} \mathrm{C}$ and to the injection pressure $(138 \mathrm{kPa})$. The high thermal conductivity of aluminum ensures a uniform temperature distribution in the mold. This means that when heating is performed from the top or bottom sides of the mold, a unidirectional heat transfer takes place through the thickness of the composite.

The mold is insulated on its lateral sides and on the top and bottom faces to minimize heat losses. Pressure and temperature sensors are placed in the mold to measure the pressure close to the injection port and vent and record the evolution of temperature in the cavity. The heating system is composed of a series of cartridges placed along the inner top and bottom surfaces of the cavity inside the two metallic parts of the mold (Figure 1). Uniform heating is applied on the top and bottom surfaces of the mold. 


\subsection{Injection System}

During the injection, compressed air pushes the resin from a container maintained under pressure to the injection gate through silicone pipes. The container is connected to compressed air by a pressure reducer equipped with a pressure gauge. Screws are used as injection port and vent in order to close the system once the injection is done.

\subsection{Instrumentation}

Three K-type thermocouples of $50 \mu \mathrm{m}$ diameter are inserted horizontally and equally spaced $(5 \mathrm{~mm})$ in the center of the mold through the thickness of the fibers. They are manufactured with a specific welding machine intended for low diameter wires. The thermocouples are slipped between the folds of the reinforcement in order to be maintained at the desired positions. Their wires are inserted through a hole bored in the frame of the mold and sealed to avoid leakage of the resin injected.

\subsection{Material and Experimental Protocol}

The resin used is the AOC T-590 preheated to $40^{\circ} \mathrm{C}$ in a bath in order to decrease its viscosity before injection. The mold and the fibers are also preheated to $40^{\circ} \mathrm{C}$. A quantity of $1.5 \%$ in mass of a catalyst of type Norox Pulcat-A is added prior to injection. The resin is degassed using a vacuum pump right before it is injected. The inlet pressure was maintained at $103 \mathrm{kPa}$ throughout the injection. All the components are assembled in the mold and the thickness of the cavity is adjusted with the screws until the desired thickness is reached. At the end of resin injection, the temperature of the mold is increased from $40^{\circ} \mathrm{C}$ to $95^{\circ} \mathrm{C}$ with a heating rate of $5^{\circ} \mathrm{C} / \mathrm{min}$ and then kept at $95^{\circ} \mathrm{C}$ during 25 minutes. The same heating rate is applied during post-cure to increase the temperature to $115^{\circ} \mathrm{C}$. Finally, compressed air through the cooling channels is used to cool the mold until ambient temperature is reached.

\section{Experimental Results}

\subsection{In-Plane Configuration}

A uniform and unidirectional thermal gradient must be applied along each planar direction of the composite specimens to determine the in-plane thermal conductivity. Hence the samples must be placed vertically so that the fibers are perpendicular to the horizontal direction of the cavity in Figure 2.

Three thermocouples are placed horizontally and equally spaced in the inplane direction, not more than $20 \mathrm{~mm}$ apart on the same horizontal plane in the center of the mold cavity. When cross-linking of the resin is completed, the specimen is removed from the mold. The central zone of the specimen, where the thermocouples are located, is cut with an electrical saw. The composite sample obtained is then placed in the cavity so that the thermocouples are positioned vertically as shown in Figure 3. Both sides of the sample are insulated with PTFE 


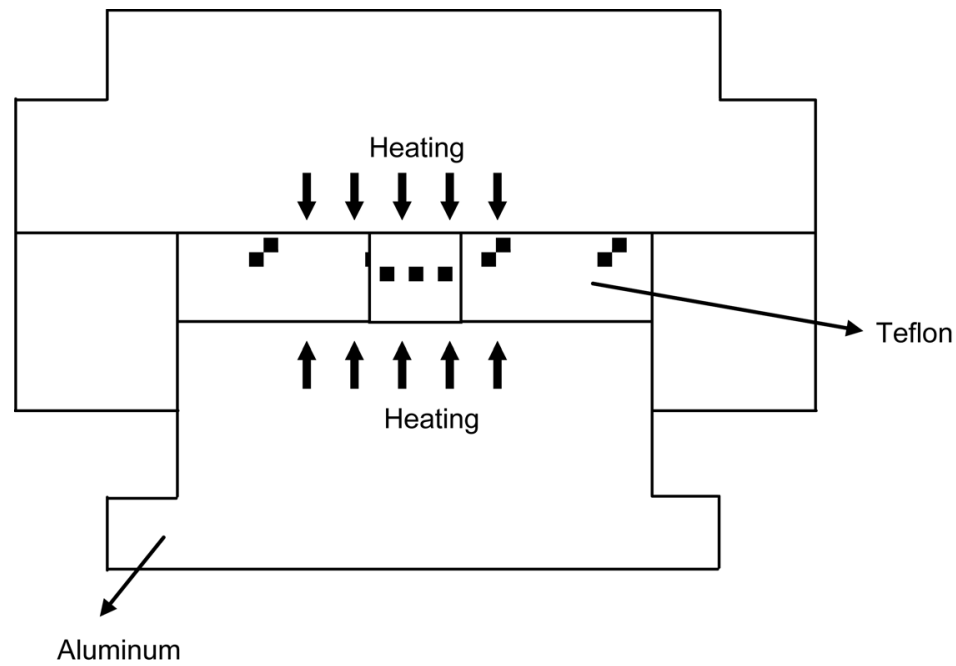

Figure 2. Schematics of the mold with the composite sample. Heated is conducted from the top and bottom surfaces.

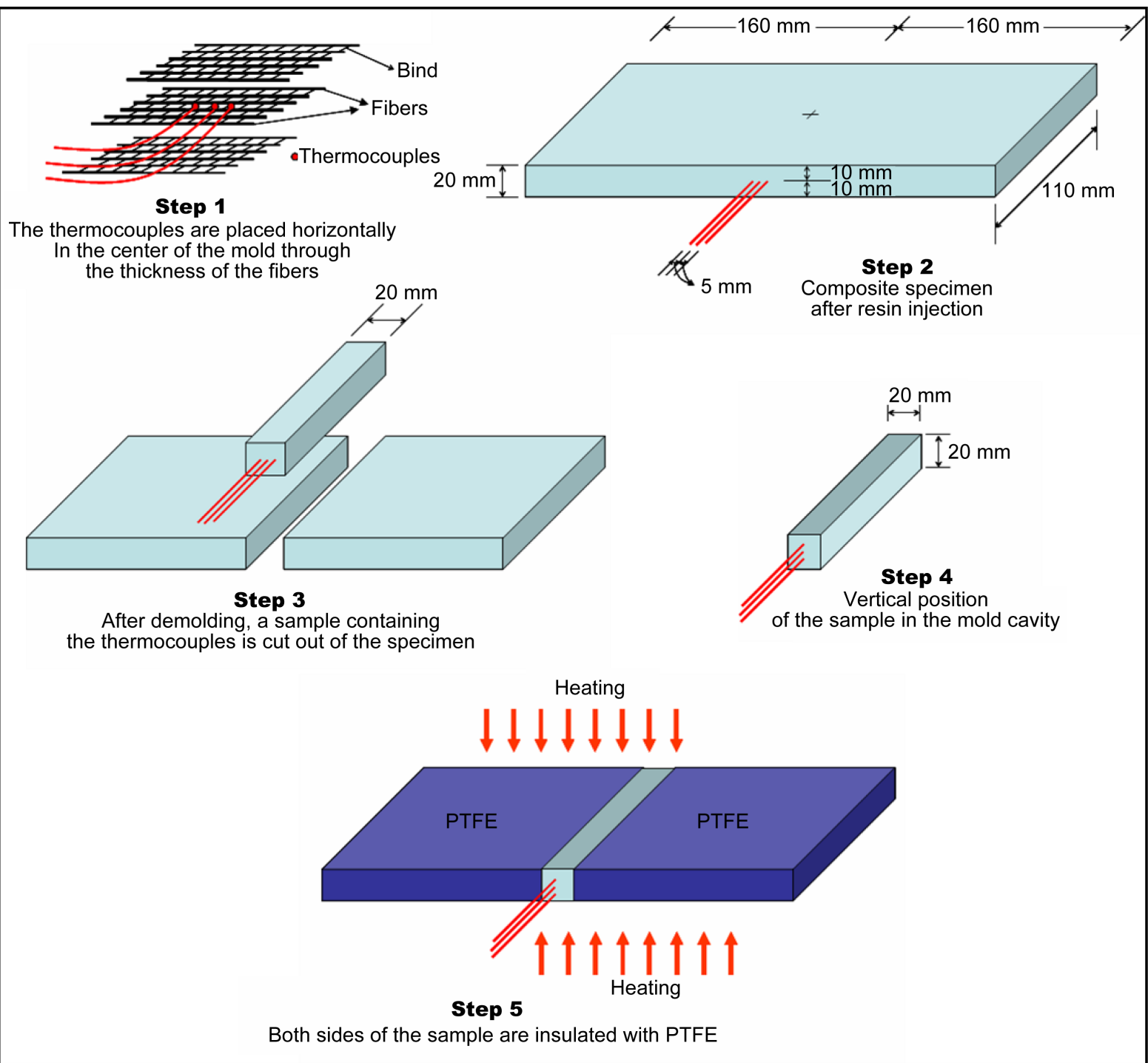

Figure 3. Set up of the composite sample with proper fiber orientation for in-plane conductivity measurements by inverse thermal analysis. 
and the mold is closed carefully to ensure that the joint separating the frame and the top plate remains in its groove. The addition of the two PTFE inserts is a relatively minor addition, but it represents here a key feature to ensure a uniform heat transfer in the in-plane directions of the composite specimen. As a matter of fact, when the composite sample, which is injected horizontally in the inplane direction, is set vertically in the test mold between the two PTFE inserts, this allows using the same mold for in-plane heat conductivity measurement as in the through-thickness direction.

To measure the in-plane thermal conductivity, the temperature of the mold is initially fixed at $30^{\circ} \mathrm{C}$ during 15 minutes to ensure a uniform isothermal state of the composite sample, and then temperature is increased to $180^{\circ} \mathrm{C}$ with a heating rate of $4^{\circ} \mathrm{C} / \mathrm{min}$. Temperature is maintained at $180^{\circ} \mathrm{C}$ during 15 minutes before the mold is cooled to the ambient temperature.

\subsection{Estimation Procedure}

Knowing the evolution of the boundary conditions in time, i.e., the top and bottom surface temperatures of the sample, the in-plane thermal conductivity will be deduced from transient temperature measurements at three given positions through the thickness of the part. As summed up in Appendix, the estimation procedure consists of minimizing a criterion based on the square difference between the measured and computed temperatures at the positions of the three thermocouples using the unidirectional (1D) conductive heat transfer equation to solve the direct problem.

To verify the unidirectional heat flow hypothesis, namely that the heat flow through the composite specimens placed in the above described configuration is unidirectional, a 2D finite element simulation of the heat transfer problem was performed. The temperature field after $2500 \mathrm{~s}$ and the mesh are shown in Figure 4. This analysis confirms that the heat flow is vertical and unidirectional in the center of the mold cavity. Heat transfer simulations were carried out with PAM$\mathrm{RTM}^{\oplus}$, a commercial finite element analysis software package dedicated to numerical simulations of the RTM process [15]. Table 1 contains the properties of the materials used in the simulations. The thickness of the cavity is set to $20 \mathrm{~mm}$ for all the numerical simulations, which is identical to the thickness of the experimental samples.

Minimization is performed by a gradient technique coupled to the adjunct variable [17]. Details on the direct problem and the estimation algorithm are given in Appendix. The temperature distribution in the mold after $2500 \mathrm{sec}$ (Figure 4) shows that heating is uniform through the thickness of the sample in the measurement area, where the thermocouples are positioned, namely in the central part of the cavity. To verify if a unidirectional heat transfer is achieved, the evolution of temperature was also compared experimentally between several positions at the same depth in the composite part by using additional thermocouples. Less than $0.5 \%$ difference was observed, which confirms that a unidirectional vertical heat transfer is effectively achieved as predicted numerically (Figure 5). 


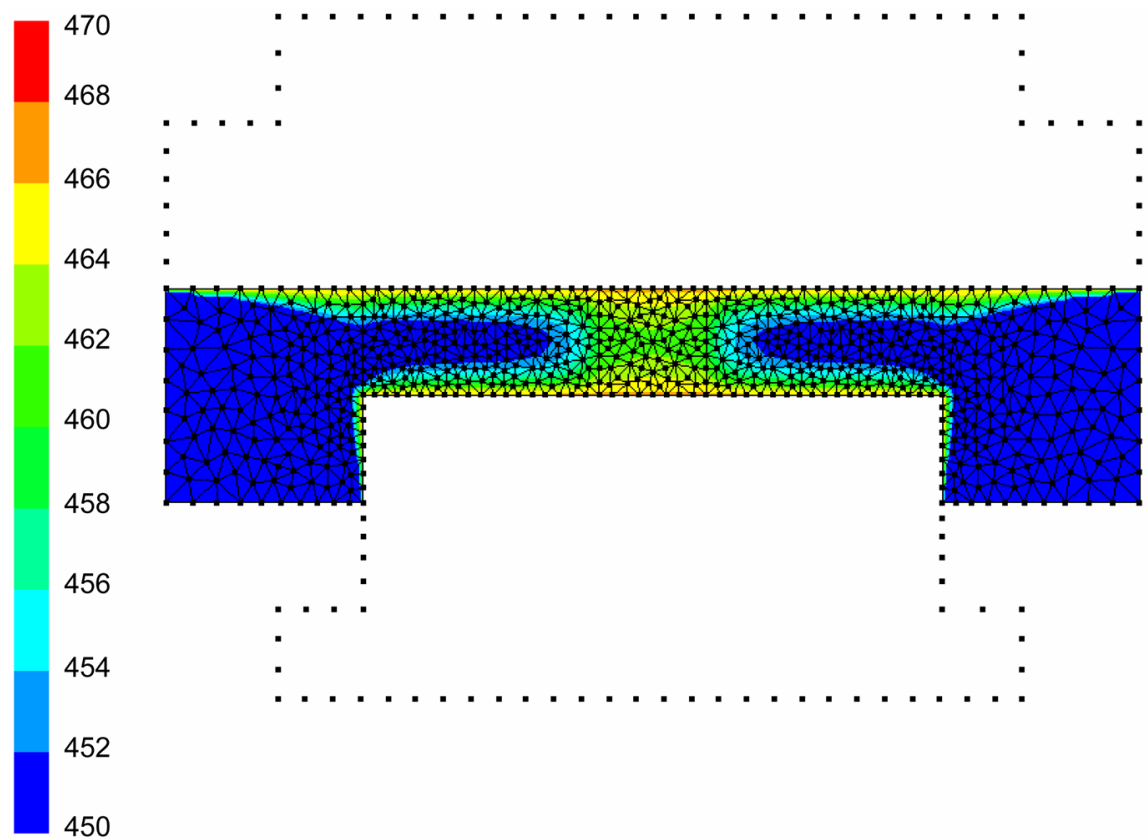

Figure 4. Temperature distribution in the mold after 2500 sec.

Table 1. Thermophysical properties of the materials used in the numerical simulation.

\begin{tabular}{cccc}
\hline & $\begin{array}{c}\text { Thermal conductivity } \\
(\mathrm{W} / \mathrm{m} \cdot \mathrm{K})\end{array}$ & $\begin{array}{c}\text { Specific heat } \\
(\mathrm{J} / \mathrm{kg} \cdot \mathrm{K})\end{array}$ & $\begin{array}{c}\text { Density } \\
\left(\mathrm{kg} / \mathrm{m}^{3}\right)\end{array}$ \\
\hline Aluminum & 237 & 900 & 2700 \\
Teflon & 0.2 & 1172 & 1450 \\
Steel & 15 & 470 & 8000 \\
Composite material & $\lambda_{/ /}$estimated & 1400 & 1835 \\
\hline
\end{tabular}

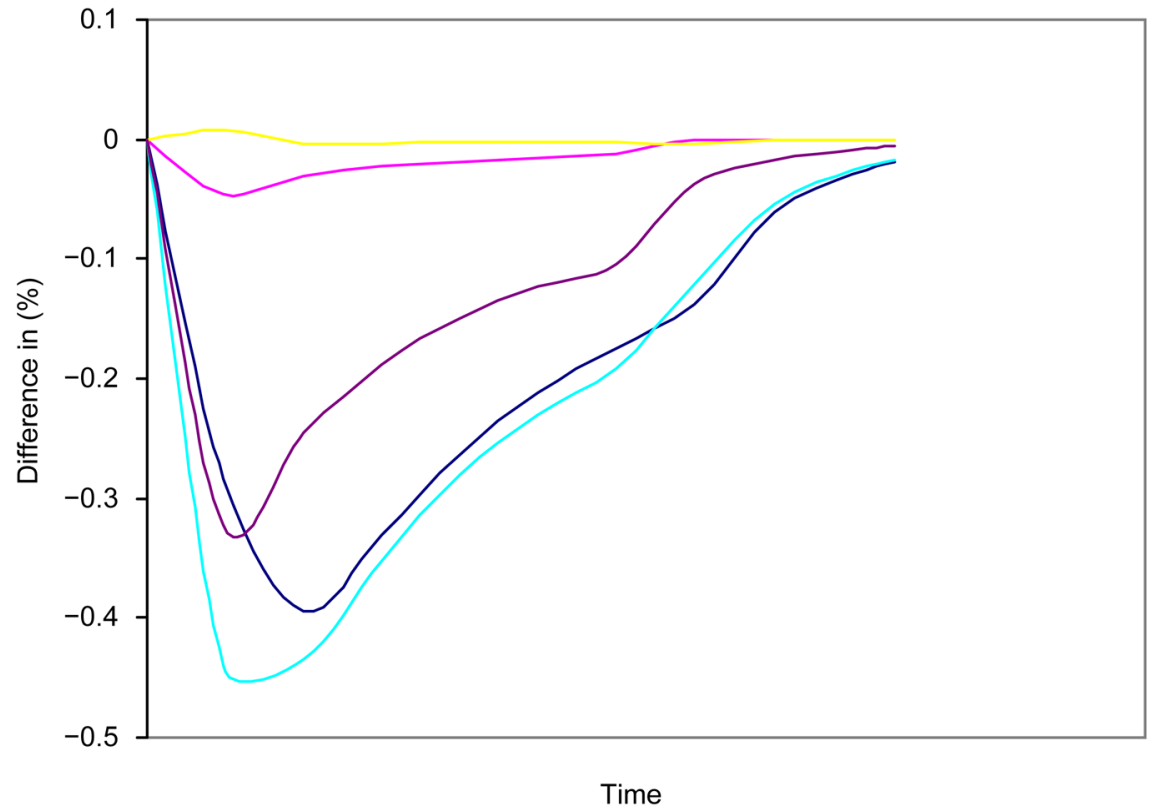

Figure 5. Evolution of temperature differences in time between thermocouples located at the same level in the composite specimen. 
The inverse methodology is implemented here for the cured composite only. However, note that the same procedure can be applied to the impregnated preform before cure. Hence the thermal conductivity $\lambda$ obtained as a function of temperature $T$ can be expressed as a function of the degree of cure $\alpha$ by applying the following rule of mixture:

$$
\lambda(T, \alpha)=(1-\alpha) \lambda_{\text {cured }}(T)+\alpha \lambda_{\text {raw }}(T)
$$

where $\lambda_{\text {raw }}(T)$ and $\lambda_{\text {cured }}(T)$ denote the time dependent thermal conductivities obtained by inverse analysis for the raw and cured composites respectively.

\subsection{Results and Discussion}

Figure 6 shows the temperature measured for the cured composite by the three thermocouples located in the center of the specimen. The two thermocouples close to the boundaries give almost the same values since they are located at the same distances from the surfaces of the mold cavity. These recorded temperatures are used as boundary conditions in the program that identifies the thermal conductivity by the inverse method. The variations of the in-plane thermal conductivity of the cured composite with temperature are displayed in Figure 7. The thermal conductivity evolves almost linearly with temperature. A linear regression gives:

$$
\lambda_{l}(T)=5 \times 10^{-5} T+0.8588(\mathrm{~W} / \mathrm{mK}) \quad(T \text { in } \mathrm{K})
$$

Note that the thermal conductivity of the cured composite can also be deduced from the thermal conductivity $\lambda_{m}$ of the resin, the thermal conductivity $\lambda_{f}$ of the fibers and the fiber volume content $v_{f}$ using the rule of mixture, all the plies being oriented at $0^{\circ}$ :

$$
\lambda_{\text {Long }}=\lambda_{m}\left(1-v_{f}\right)+\lambda_{f} v_{f}
$$

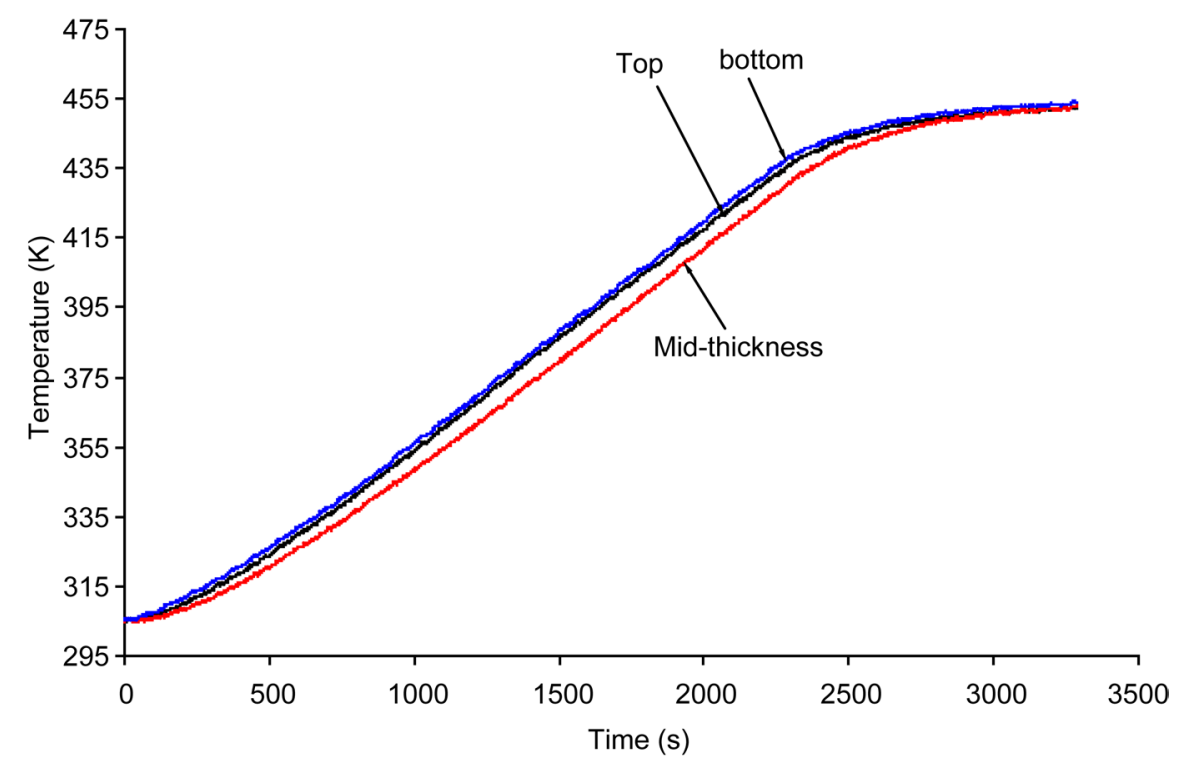

Figure 6. Temperature evolution of the three thermocouples positioned along the inplane direction of the cured composite during heating. 


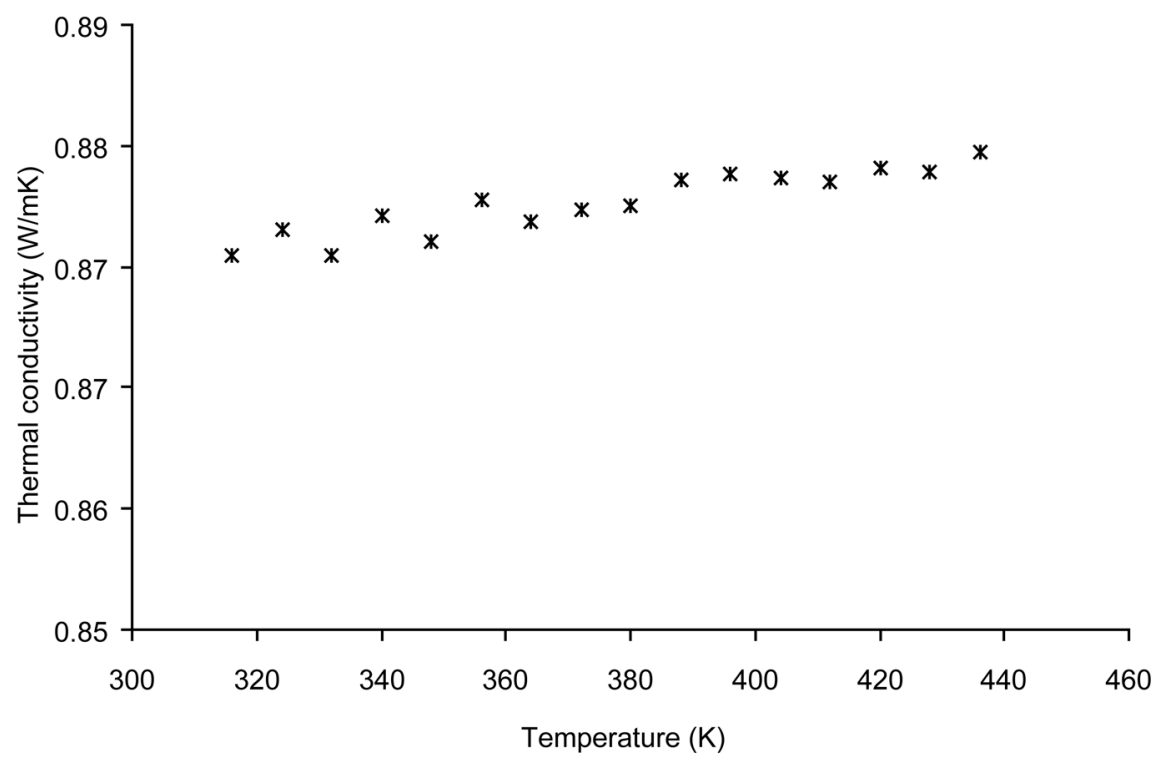

Figure 7. Evolution with temperature of the in-plane thermal conductivity of the cured composite.

Knowing the superficial density $\rho_{\text {surf }}\left(\mathrm{kg} \cdot \mathrm{m}^{-2}\right)$ of each layer in the stack, the number of layers $\mathrm{n}$ introduced in the stack, the volumetric density $\rho_{v o l}\left(\mathrm{Kg} \cdot \mathrm{m}^{-3}\right)$ of the fibers and the thickness $h(m)$ of the stack, the fiber volume content is determined by the following equation:

$$
v_{f}=\frac{\rho_{\text {surf }} \times n}{\rho_{\text {vol }} \times h} \times 100
$$

The fiber volume content for all the experiments performed is equal to $61 \%$. In order to apply the rule of mixture, the thermal conductivity of the cured resin was also measured. A small piece made of the same polyester resin is used to hold with adhesive the thermocouples at given positions across the thickness of the cavity during the injection. The sample is placed in the center of the mold where the thermal conductivity is to be identified. The resin is then injected and the thermocouples are impregnated by the resin. After cure of the resin, the same experimental protocol followed to acquire temperature data for the cured composite is used. Figure 8 shows the temperature distribution in the thickness of the cured resin. The temperatures recorded allow to identify the thermal conductivity by inverse analysis. The results of Figure 9 give after estimation a variation of thermal conductivity with temperature of about $5 \%$.

Considering the thermal conductivity of glass fibers to be roughly equal to $\lambda_{f}=$ $1.3 \mathrm{~W} / \mathrm{mK}[12]$, the thermal conductivity of the composite can be estimated using Equation (3) and compared to the experimental value. The comparison between the experimental in-plane thermal conductivity of the cured composite and the one derived by the rule of mixture is shown in Figure 10. A difference of less than $0.5 \%$ is observed between the experiment and the model. This validates the consistency of the proposed approach based on inverse analysis to measure the in-plane heat conductivity of composites. 


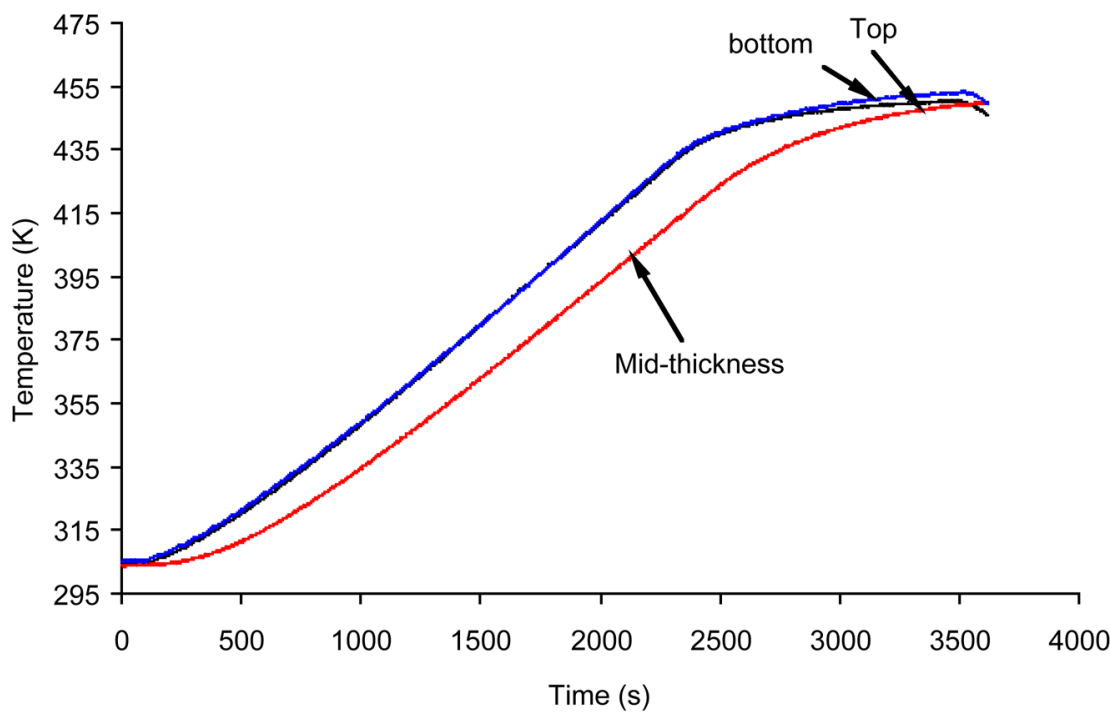

Figure 8. Temperature evolution of the three thermocouples through the thickness of the cured resin during heating.

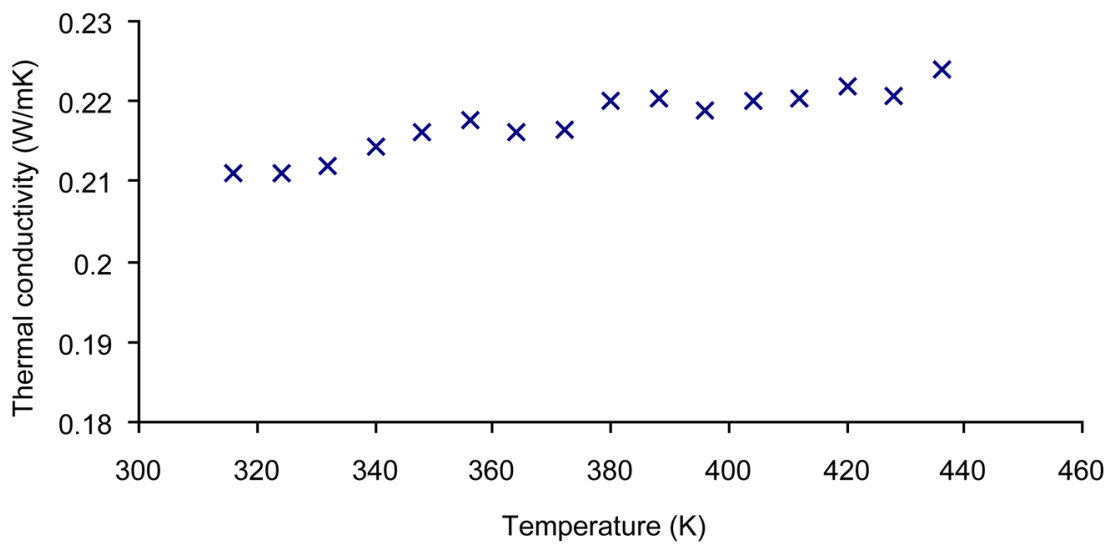

Figure 9. Evolution of the thermal conductivity of the cured resin with temperature.

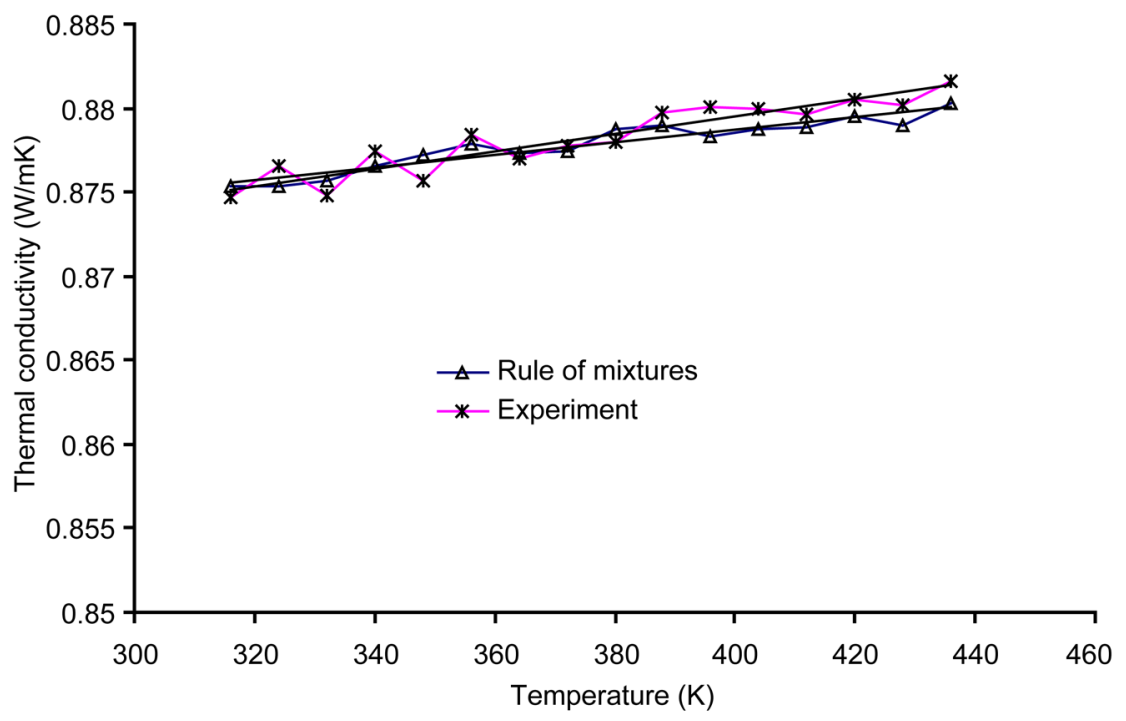

Figure 10. Comparison between the experimental in-plane thermal conductivity of the cured composite and the value derived from the rule of mixture. 


\section{Conclusions}

An inverse thermal analysis was conducted to determine the in-plane thermal conductivity of composite specimens. This paper shows how the same mold devised initially for through-thickness measurements can be successfully used to measure the in-plane thermal conductivity of composites. The mold has an adjustable cavity depth and is equipped with heating cartridges and cooling channels to control temperature. Two pressure sensors monitor the pressure during the injection. Numerical simulations were carried out to verify that a unidirectional heat transfer takes place in the cavity during the experiments.

An experimental protocol was followed to inject planar composite specimens. A sample is cut out of the cured composite to measure the in-plane thermal conductivity. Heating is applied to the sample and the evolution of temperature in time is recorded using the three thermocouples previously positioned along the length of the composite sample. The in-plane thermal conductivity is then estimated by inverse thermal analysis. The same experimental procedure and inverse analysis algorithm are used to measure the thermal conductivity of the cured resin. Knowing the thermal conductivity of glass fibers, the rule of mixture allows calculating the thermal conductivity of the composite. Calculated values differ by less than $0.5 \%$ from experimental results. This demonstrates the consistency of the proposed measurement technique based on inverse thermal analysis.

The thermal conductivity in the case studied here varies only slightly with temperature. For the polyester resin used in this investigation, the proposed methodology shows that the insulating properties are relatively stable with temperature with only a 5\% upward drift as temperature increases. The thermal conductivity of most commercial resins is available in the literature. In general, the thermal conductivity of composites is not known beforehand. Therefore, it is useful to develop a general methodology and design an experimental test mold to measure the in-plane heat conductivity as a function of temperature in a single experiment. Thanks to inverse analysis, the same time is required to conduct the proposed experiment and obtain the heat conductivity as a function of temperature as to perform a single thermal conductivity measurement at any given temperature by other techniques.

\section{Acknowledgements}

The contributions of the National Science and Engineering Research Council of Canada (NSERC) and Fonds Québécois de Recherche sur la Nature et la Technologie (FQRNT) are gratefully acknowledged.

\section{References}

[1] Assaf, B., Menge, H., Sobotka, V. and Trochu, F. (2005) Development of a Characterization Mold to Measure the Transverse Thermal Conductivity of a Composite Material by Inverse Analysis. Journal of Reinforced Plastics and Composites, 24, 1837-1854. https://doi.org/10.1177/0731684405052808

[2] Tardif, X., Agazzi, A., Sobotka, V., Boyard, N., Jarny, Y. and Delaunay, D. (2012) A 
Multifunctional Device to Determine Specific Volume, Thermal Conductivity and Crystallization Kinetics of Semi-Crystalline Polymers. Polymer Testing, 31, 819827. https://doi.org/10.1016/j.polymertesting.2012.05.008

[3] Villiere, M., Lecointe, D., Sobotka, V., Boyard, N. and Delaunay, D. (2013) Experimental Determination and Modeling of Thermal Conductivity Tensor of Carbon/Epoxy Composite. Composites Part A: Applied Science and Manufacturing, 46, 60-68. https://doi.org/10.1016/j.compositesa.2012.10.012

[4] Goodson, K.E. and Flik, M.I. (1994) Solid Layer Thermal-Conductivity Measurement Techniques. Applied Mechanics Reviews, 47, 101. https://doi.org/10.1115/1.3111073

[5] Rides, M., Morikawa, J., Halldahl, L., Hay, B., Lobo, H., Dawson, A. and Allen, C. (2009) Intercomparison of Thermal Conductivity and Thermal Diffusivity Methods for Plastics. Polymer Testing, 28, 480-489.

https://doi.org/10.1016/j.polymertesting.2009.03.002

[6] Wakeham, W.A. and Assael, M.J. (1999) Thermal Conductivity Measurement. In: Webster, J.G., Ed., Instrumentation and Sensors Handbook, CRC Press, Boca Raton, Florida. https://doi.org/10.1201/9780415876179.ch33

[7] ASTM E1530-06, Standard Test Method for Evaluating the Resistance to Thermal Transmission of Materials by Guarded Heat Flow Meter Technique.

[8] Norme ISO 8894-1, Matériaux réfractaires-Détermination de la conductivité thermique, 1984.

[9] Hay, B., Filtz, J.R., Hameury, J. and Rongione, L. (2005) Uncertainty of Thermal Diffusivity Measurements by Laser Flash Method. International Journal of Thermophysics, 26, 1883-1898. https://doi.org/10.1007/s10765-005-8603-6

[10] ISO 22007-4:2008-Plastics-Determination of Thermal Conductivity and Thermal Diffusivity-Part 4: Laser Flash Method (2014).

http://www.iso.org/iso/catalogue_detail.htm?csnumber $=42047$

[11] Gustavsson, M., Karawacki, E. and Gustafsson, S.E. (1994) Thermal Conductivity, Thermal Diffusitvity and Specific Heat of Thin Samples from Transient Measurement with Hot Disk Sensors. Review of Scientific Instruments, 65, 3856. https://doi.org/10.1063/1.1145178

[12] ISO 22007-2:2008-Plastics-Determination of thermal Conductivity and Thermal Diffusivity-Part 2: Transient Plane Heat Source (Hot Disc) Method. http://www.iso.org/iso/catalogue_detail.htm

[13] Shen, B., Zeng, Z., Lin, C. and Hu, Z. (2013) Thermal Conductivity Measurement of Amorphous $\mathrm{Si} / \mathrm{SiGe}$ Multilayer Films by 3 Omega Method. International Journal of Thermal Sciences, 66, 19-23. https://doi.org/10.1016/j.ijthermalsci.2012.10.022

[14] Hashimoto, T., Morikawa, J., Kurihara, T. and Tsuji, T. (1997) Frequency Dependent Thermal Diffusivity of Polymers by Temperature Wave Analysis. Thermochimica Acta, 304-305, 151-156. https://doi.org/10.1016/S0040-6031(97)00026-9

[15] ESI-Group. PAM-RTM Simulation Software of the Resin Transfer Molding (RTM) Process. https://www.esi-group.com/

[16] Chen, H., Ginzburg, V.V., Yang, J., Yang, Y., Liu, W., Huang, Y., Du, L. and Chen, B. (2016) Thermal Conductivity of Polymer-Based Composites: Fundamentals and Applications. Progress in Polymer Science, 59, 41-85. https://doi.org/10.1016/j.progpolymsci.2016.03.001

[17] Woodbury, K.A. and Jarny, Y. (2003) Inverse Engineering Handbook-The Adjoint Method to Compute the Numerical Solutions of Inverse Problems. C. Press LLC. 


\section{Appendix: Description of the Inverse Methodology}

\section{A1. Direct Problem}

The direct problem consists of solving numerically the one-dimensional transient heat conduction equation. The energy balance expressed in terms of temperature $\mathrm{T}$ writes as follows:

$$
\frac{\partial}{\partial x}\left[\lambda(T) \frac{\partial T}{\partial x}\right]=\rho C_{p}(T) \frac{\partial T}{\partial t} ; \quad 0<x<L
$$

where the following parameters are defined for the composite material:

$\rho$ : density $\left(\mathrm{kg} / \mathrm{m}^{3}\right)$,

$C_{p}(T):$ specific heat $\left.(\mathrm{J} / \mathrm{jgK})\right)$,

$\lambda(T)$ : thermal conductivity $(\mathrm{W} / \mathrm{mK})$,

$L$ : length of the part (m),

The governing partial differential equation of heat transfer (2) is subjected here to the following boundary conditions:

$$
\begin{cases}T(0, t)=T_{1}(t) & t>0 \\ T(L, t)=T_{2}(t) & t>0 \\ T(x, 0)=T_{0}(x) & 0<x<L\end{cases}
$$

where $T_{1}(t)$ and $T_{2}(t)$ denote the temperatures at the top and the bottom boundaries of the part, while $T_{0}(x)$ represents the initial temperature field. The temperature dependent specific heat $C_{p}(T)$ and the constant density $\rho$ are known. The specific heat is determined here as a function of temperature by differential scanning calorimetry, so only the thermal conductivity $\lambda(T)$ of the composite needs to be identified in Equation (A1).

\section{A2. Inverse Algorithm}

For a number $N$ of thermocouples (here $N=3$ ) set in the cavity, the least square criterion $J(\lambda)$ is expressed as:

$$
J(\lambda)=\sum_{j=1}^{N} \int_{t=0}^{t_{F}}\left[T\left(x_{j}, t\right)-Y_{j}(t)\right]^{2} \mathrm{~d} t
$$

where $t_{f}$ is the total time of the experiment, $Y_{j}(t)$ represents the temperature measured at point $x_{j} 1 \leq j \leq N$, and $T\left(x_{j}, t\right)$ the temperature calculated at the same locations. The direct problem with an initial value of $\lambda$ for the composite is solved numerically. The difference between the calculated and experimentally measured temperatures is estimated at each location. The functional $J(\lambda)$ of Equation (A3) is minimized, subjected to the constraint represented by the ordinary differential equation of heat conduction (A1). As described in [17], the thermal conductivity minimizing $J(\lambda)$ is identified by a gradient descent method with Lagrange multipliers to account for the differential Equation (A1) as constraint. 
Submit or recommend next manuscript to SCIRP and we will provide best service for you:

Accepting pre-submission inquiries through Email, Facebook, LinkedIn, Twitter, etc. A wide selection of journals (inclusive of 9 subjects, more than 200 journals)

Providing 24-hour high-quality service

User-friendly online submission system

Fair and swift peer-review system

Efficient typesetting and proofreading procedure

Display of the result of downloads and visits, as well as the number of cited articles Maximum dissemination of your research work

Submit your manuscript at: http://papersubmission.scirp.org/

Or contact ojcm@scirp.org 